\title{
Magnetic microparticle-based multimer detection system for the detection of prion oligomers in sheep
}

\author{
Kuntaek Lim' \\ Su Yeon Kim² \\ Byoungsub Lee' \\ Christiane Segarra ${ }^{3}$ \\ Sungmin Kang' \\ Youngran $\mathrm{Ju}^{2}$ \\ Mary Jo Schmerr ${ }^{4}$ \\ Joliette Coste ${ }^{3}$ \\ Sang Yun Kim ${ }^{5}$ \\ Takashi Yokoyama ${ }^{6}$ \\ Seong Soo $\mathrm{A} \mathrm{An}^{7}$ \\ 'Department of Research and \\ Development, PeopleBio Inc., \\ ${ }^{2}$ Department of Arborbiology, \\ Korean Center for Diseases and \\ Control (KCDC), Seoul, Republic \\ of Korea; ${ }^{3}$ Department of Blood \\ Screening, Etablissement Français \\ Du Sang (EFS), Montpellier, France; \\ ${ }^{4}$ Ames Laboratories, US Department \\ of Energy (USDOE), lowa State \\ University, Ames, IA, USA; \\ ${ }^{5}$ Department of Neurology, Seoul \\ National University Bundang Hospital, \\ Sungnam-si, Republic of Korea; \\ ${ }^{6}$ Department of Prion Research, \\ National Institute of Animal Health, \\ Tsukuba, Japan; ${ }^{7}$ Department \\ of Bionano Technology, Gachon \\ University, Sungnam-si, Republic \\ of Korea
}

Correspondence: Seong Soo A An

Department of Bionano Technology,

Gachon Medical Research Institute,

Gachon University, Sungnam-si 46I-70I,

Gyeonggi-do, Republic of Korea

Tel/fax +82 3I 7508755

Email seongaan@gachon.ac.kr
This article was published in the following Dove Press journal:

International Journal of Nanomedicine

9 September 2015

Number of times this article has been viewed

Abstract: Transmissible spongiform encephalopathies (TSEs) are zoonotic fatal neurodegenerative diseases in animals and humans. TSEs are commonly known as bovine spongiform encephalopathy in cattle, scrapie in sheep and goats, chronic wasting disease in cervids, and Creutzfeldt-Jakob disease in humans. The putative transmissible agents are infectious prion proteins $\left(\mathrm{PrP}^{\mathrm{Sc}}\right)$, which are formed by the conversion of the normal prion protein on the glycoprotein cell surface in the presence of other $\operatorname{PrP}^{\mathrm{Sc}}$. Reports of the transmission of TSEs through blood raised considerable concern about the safety of blood and blood products. To address this issue, many laboratories attempted to develop a sensitive and accurate blood diagnostic test to detect $\mathrm{PrP}^{\mathrm{Sc}}$. Previously, we reported that, compared to normal controls, the multimer detection system (MDS) was more efficient in detecting $\operatorname{PrP}^{\mathrm{Sc}}$ in infected hamster brain homogenate, mouse plasma spiked with purified $\operatorname{PrP}^{\mathrm{Sc}}$ from scrapie mouse brain, and scrapie-infected hamster plasmas. MDS differentiates prion multimers from the cellular monomer through the multimeric expression of epitopes on prion multimers, in contrast to the monomeric form. In this study, MDS detected $\mathrm{PrP}^{\mathrm{Sc}}$ in plasma samples from scrapie-infected sheep expressing clinical symptoms, demonstrating $100 \%$ sensitivity and specificity in these samples. Plasma samples from asymptomatic lambs at the preclinical stage (8-month-old naturally infected offspring of scrapie-infected parents expressing a highly susceptible genotype) tested positive with $50 \%$ sensitivity and $100 \%$ specificity. In the first of two coded analyses using clinical scrapie-infected sheep and normal healthy samples, MDS successfully identified all but one of the clinical samples with $92 \%$ sensitivity and $100 \%$ specificity. Similar results were obtained in the second coded analysis using preclinical samples. MDS again successfully identified all but one of the samples with $87 \%$ sensitivity and $100 \%$ specificity. The false-negative sample was subjected to a protease pretreatment. In conclusion, MDS could accurately detect scrapie in plasma samples at both preclinical and clinical stages. From these studies, we conclude that MDS could be a promising tool for the early diagnosis of TSEs from blood samples.

Keywords: transmissible spongiform encephalopathies, infectious prion proteins, normal prion protein, sensitivity, specificity, biomarker

\section{Introduction}

Transmissible spongiform encephalopathies (TSEs) are transmitted through blood or organ transplantation, and can cross the species barrier much more easily than previously thought. ${ }^{1}$ Infectious prion proteins $\left(\mathrm{PrP}^{\mathrm{Sc}}\right)$ from other species can be introduced into mice and are replicated in the spleen without brain involvement. ${ }^{1}$ The putative transmissible agents are $\mathrm{PrP}^{\mathrm{Sc}}$, which are formed by the conversion of the normal prion protein $(\mathrm{PrP})$ on the glycoprotein cell surface in the presence of other $\mathrm{PrP}^{\mathrm{Sc}}$. They propagate, recruit, and convert the normal protease-sensitive PrP into additional misfolded 
and aggregated $\mathrm{PrP}^{\mathrm{Sc}}$. The lack of prion-specific nucleic acids, polypeptides, or immune responses has prevented the use of conventional detection methods for detecting this pathogen. $\mathrm{PrP}^{\mathrm{Sc}}$ have been used as the main biomarker and criteria for diagnosing TSEs. Tissues from the brain and lymphoid organs, such as tonsils, spleen, and lymphatic system, have been used for the detection of proteinase $\mathrm{K}(\mathrm{PK})$-resistant $\mathrm{PrP}^{\mathrm{Sc}}$ (PrPres) by immunohistochemistry (IHC), Western blot, or enzyme-linked immunosorbent assay (ELISA). ${ }^{2}$ Many of the current detection tests exploit elevated PrPres expression, digestion patterns, or the conformational stability of $\mathrm{PrP}^{\mathrm{Sc}}$ to distinguish $\mathrm{PrP}^{\mathrm{Sc}}$ from non-disease-inducing normal PrP. ${ }^{3}$ Bodily fluids, including blood, saliva, and nasal fluids, also demonstrate infectivity, and several assays have been developed to test for $\mathrm{PrPsc}^{\mathrm{Sc}}$ in these samples, ${ }^{4-7}$ with variable success. ${ }^{8}$ Although blood is considered the ideal choice for diagnosis due to the minimally invasive collection method, the current methods used for the detection of the protein in blood samples, which involve protease digestion, could destroy the more fragile $\mathrm{PrPsc}^{\mathrm{Sc}}$, thus resulting in an inaccurate result. ${ }^{9}$ This could be problematic, since the most infectious particles of PrPs were shown to exist as relatively smaller molecular masses. ${ }^{10}$ Due to a limited understanding of the properties of $\mathrm{PrP}^{\mathrm{Sc}}$ in the blood, several restrictions have been imposed on blood donation and the safety of blood-derived products, ${ }^{11}$ which has increased the cost of these products. Reports of the transmission of both scrapie and bovine spongiform encephalopathy (BSE) by blood transfusion into sheep ${ }^{12}$ and four cases of transmission of variant CreutzfeldtJakob disease (vCJD) through blood transfusions ${ }^{13}$ heightened concerns about the safety of blood and blood products. ${ }^{12}$ Another report suggested that the prevalence of subclinical vCJD infections could be as high as one in 4,000 people in certain UK cohorts. ${ }^{14}$ The long incubation period for vCJD raised concerns about the possibility of disease transmission by asymptomatic carriers of $\mathrm{PrP}^{\mathrm{Sc}}$, who could silently transmit the disease through blood donation/transfusions, organ transplants, dental procedures, or surgeries..$^{15}$

Prion decontamination is exceptionally difficult due to the protease resistance and stability of $\mathrm{PrP}^{\mathrm{Sc}}$. Pre-symptomatic detection of prion infection in individuals or animals could prevent transmission and generate incentives to develop effective treatments. Protein misfolding cyclic amplification (PMCA) ${ }^{16}$ and enhanced quaking-induced protein conversion ${ }^{16}$ assays are sensitive enough to detect low levels of $\mathrm{PrP}^{\mathrm{Sc}}$ in the blood; however, the processing times for these assays are very long. There is, therefore, still a need for quick and accurate TSE screening tests that provide results in hours rather than days. ${ }^{16}$
The central event in TSEs is the conformational transition of PrP from a monomer into a higher beta-sheet containing multimers or aggregates. We therefore reasoned that it might be possible to establish a sufficiently sensitive screening method for the detection of $\mathrm{PrP}^{\mathrm{Sc}}$ in blood by utilizing the multimeric feature of $\mathrm{PrP}^{\mathrm{Sc}}$, instead of protease digestion or amplification, without limiting its potential to be combined with other methods. Multimer detection system (MDS) was previously reported to differentiate brain and blood samples of scrapie-infected hamsters and mice from those of respective age-matched normal controls. ${ }^{17}$ MDS utilizes an epitope-overlapping antibody system to differentiate multimers of $\mathrm{PrP}^{\mathrm{Sc}}$ from the normal monomer in the blood by inducing competition against a unique epitope, where multiple copies would exist in multimers and only one would exist in the monomeric form (Figure 1).

The following combinations of sheep $\mathrm{PrP}^{\mathrm{Sc}}$ can create diverse homozygote and heterozygote genotypes: Ala/Val at codon 136, Arg/His at codon 154, and Arg/Gln at codon 171. ARR/ARR (Ala/Arg/Arg) would be least susceptible to scrapie transmission, whereas VRQ/VRQ (Val/Arg/Gln) would be the most susceptible genotype. Other genotypes of various combinations could have varying degrees of susceptibility. Here, MDS was used to test the plasmas from eleven clinical stage scrapie-infected sheep with natural and experimental infections of diverse genotypes, and 22 normal controls. We next tested the plasma samples of scrapie-infected sheep at the preclinical stage ( 8 months) that were infected with highly susceptible genotypes, and compared the results with those of the normal controls. Finally, two blinded panels of clinical and preclinical samples from scrapie-infected animals were coded and tested by MDS, and compared to those of normal healthy samples.

\section{Materials and methods Healthy samples}

Normal plasma samples were numbered $12-33$ for the purpose of this study. Normal plasma \#12 was obtained from Ames Laboratories (US Department of Energy, Ames, IA, USA) and had the ARQ/ARQ genotype. It was drawn from asymptomatic healthy sheep, identified among the scrapiefree flock, in ethylenediaminetetraacetate (EDTA) tubes. Normal sheep plasmas in citrate tubes, obtained from Innovative Research (Novi, MI, USA), were used as the normal pooled sample (\#13). Other normal samples (\#14-20) were obtained from the National Institute of Animal Health 


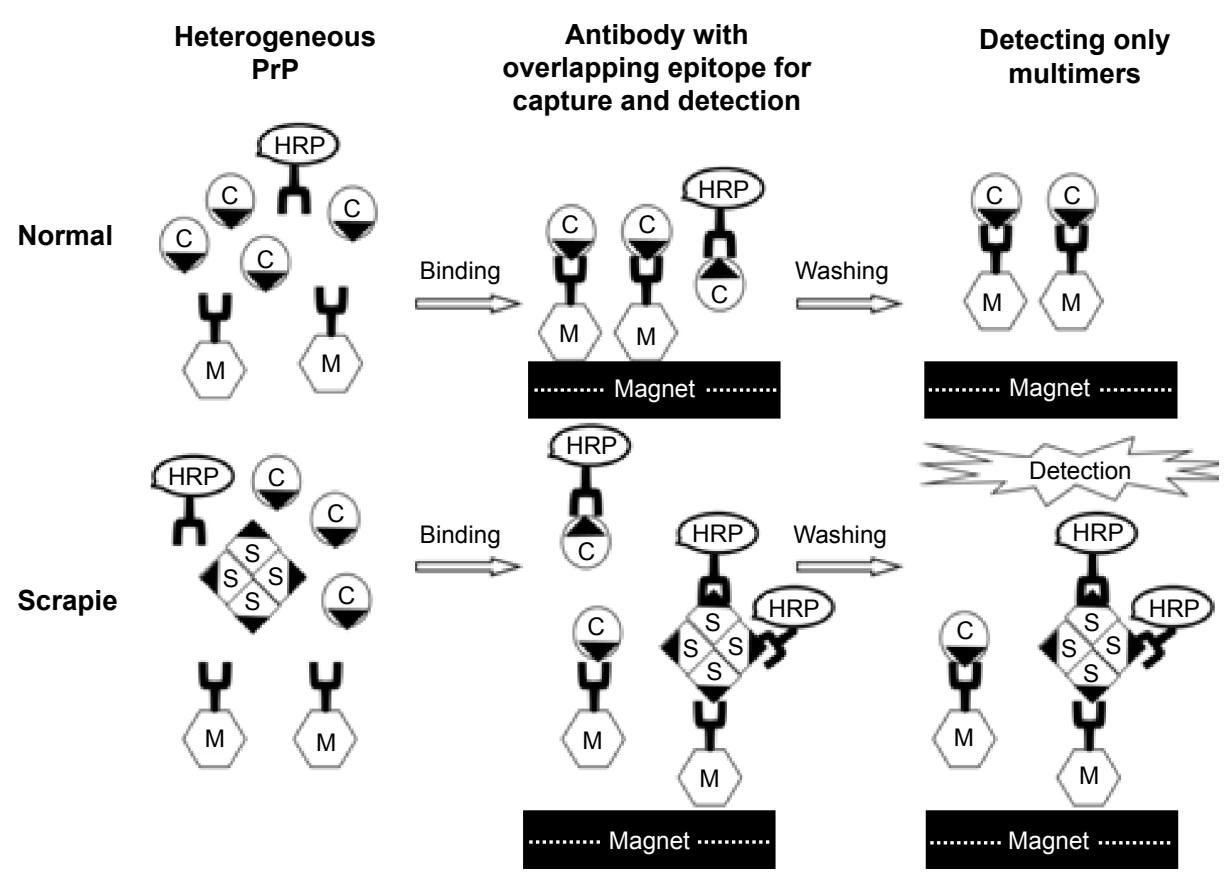

Figure I Schematic diagram of the MDS.

Notes: The overall experimental procedure of MDS is described in the following steps: I) incubation of plasma sample with both capture and HRP-conjugated detection antibodies, 2) pull-down of magnetic beads with a magnet, and 3) washes and detection with a chemiluminescent substrate. Letters, $C$ and $M$, in the figure represent the normal (control) and multimeric forms of PrPs, respectively.

Abbreviations: MDS, multimer detection system; HRP, horse radish peroxidase; PrP, prion protein.

(NIAH, Tsukuba, Japan), and were all of the ARQ/ARQ genotype. Brain sections were analyzed by IHC at NIAH and showed no spongiform. Additional plasma samples from normal healthy flock without any neurological symptoms were drawn in citrated tubes, without genotype analysis. These were obtained from the Hungary Academy of Sciences, Budapest, Hungary (\#21-27) and the Korean Center for Diseases and Control, Daejeon, Chungcheongbuk-do, South Korea (\#28-33).

\section{Clinical samples}

Clinical scrapie sheep plasma samples (\#1-11) were obtained from Baltimore Research and Education Foundation, Inc. (BREF, University of Maryland, College Park, MD, USA) and Ames Laboratories as indicated in Table 1. Postmortem diagnosis of scrapie samples from Ames Laboratories and BREF was made by biopsy of the rectal tissues, postmortem lymphoid tissues (retropharyngeal lymph nodes, palatine tonsils, spleen, ileocecal junction, ileocecal lymph nodes,

Table I IHC detection of PrPSc in tissues from scrapie-infected sheep with clinical symptoms

\begin{tabular}{|c|c|c|c|c|c|c|c|c|c|c|c|c|}
\hline \multicolumn{2}{|c|}{ Sample \# } & \multirow{2}{*}{$\begin{array}{l}\text { Origin } \\
\text { Individual }\end{array}$} & \multirow{2}{*}{$\begin{array}{l}\text { Genotype } \\
\text { AVQQ }\end{array}$} & \multirow{2}{*}{$\begin{array}{l}\text { Plasma } \\
\mathrm{P}\end{array}$} & \multirow{2}{*}{$\begin{array}{l}\text { Infectivity } \\
\text { Experimental }\end{array}$} & \multirow{2}{*}{$\begin{array}{l}\text { Anticoagulant } \\
A C D\end{array}$} & \multirow{2}{*}{$\begin{array}{l}\text { Third eyelid } \\
\mathrm{P}\end{array}$} & \multirow{2}{*}{$\begin{array}{l}\text { Tonsil } \\
\mathrm{P}\end{array}$} & \multirow{2}{*}{$\begin{array}{l}\text { Mand } \\
P\end{array}$} & \multirow{2}{*}{$\begin{array}{l}\text { Retro } \\
P\end{array}$} & \multirow{2}{*}{$\begin{array}{l}\text { Mess } \\
\mathrm{P}\end{array}$} & \multirow{2}{*}{$\frac{\text { Brain }}{W P}$} \\
\hline $\mathrm{I}$ & UMD & & & & & & & & & & & \\
\hline 2 & & Individual & AVQQ & $\mathrm{P}$ & Natural & $A C D$ & $\mathrm{P}$ & $P$ & $\mathrm{P}$ & $\mathrm{P}$ & $P$ & $P$ \\
\hline 3 & & Individual & AAQQ & SP & Experimental & $A C D$ & $P$ & $P$ & $P$ & $\mathrm{P}$ & $P$ & $P$ \\
\hline 4 & & Pooled & & $P$ & Natural & $A C D$ & & & & & & \\
\hline 5 & & Pooled & & $P$ & Experimental & $A C D$ & & & & & & \\
\hline 6 & Ames & Individual & & $P$ & Natural & EDTA & & & & & & $P$ \\
\hline 7 & & Individual & & $\mathrm{SP}$ & Natural & EDTA & & & & & & $P$ \\
\hline 8 & & Individual & & WP & Natural & EDTA & & & & & & $P$ \\
\hline 9 & & Individual & AAQQ & WP & Natural & EDTA & & $\mathrm{SP}$ & & & & SP \\
\hline 10 & & Individual & AAQQ & $P$ & Natural & EDTA & & $P$ & & & & $P$ \\
\hline 11 & & Individual & AAQQ & $P$ & Natural & EDTA & & $P$ & & & & $P$ \\
\hline
\end{tabular}

Notes: Sheep positive for PrPsc accumulation in the examined tissues are indicated. PrPsc-labeling intensity is indicated as negative (-), minimal to slight (WP), moderate (P), or strong (SP).

Abbreviations: PrPsc, infectious prion proteins; IHC, immunohistochemistry; EDTA, ethylenediaminetetraacetate; UMD, University of Maryland; ACD, acid citrate dextrose. 
and mesenteric lymph nodes), and brains, and $\mathrm{PrP}^{\mathrm{Sc}}$ detection by IHC in sheep with clinical signs. As indicated in Table 1, plasma samples were collected from both naturally and intracerebrally transmitted lamb with either EDTA or citrated anticoagulants. Tissues were fixed in formalin and processed according to standard procedures.

\section{Preclinical samples}

Ten preclinical and eight normal sheep plasmas were obtained from Ecole Nationale Vétérinaire (Toulouse, France) and Etablissement Français Du Sang (Montpellier, France). All preclinical samples were genotype-matched for the highly susceptible VRQ/VRQ genotype. All parent ewes and rams of the ten preclinical sheep showed scrapie symptoms after 22 months and died of scrapie. The preclinical plasmas were drawn from lambs, predisposed to scrapie, at the age of 8 months, as previously reported. ${ }^{18}$ An antemortem diagnosis was provided by IHC analysis of their tonsils, which showed $\mathrm{PrP}^{\mathrm{Sc}}$ deposition at 3 months of age, as previously reported, indicating the progression of the disease at 8 months. All preclinical lambs came down with scrapie at the age of 22-24 months.

\section{Coded samples}

A blinded panel of 12 coded plasma samples from scrapieinfected sheep showing clinical symptoms and 21 normal healthy controls was made available. These plasma samples from well-characterized clinical sheep were donated by BioRad Inc. (Paris, France). Citrate was used as an anticoagulant. All clinical scrapie samples along with normal control samples were coded independently and tested by MDS. After acquiring the data, the key was opened, and the results were analyzed. Another panel of seven preclinical and 15 normal sheep plasmas for the coded test was received as a gift from IDEXX Inc. (Westbrook, MA, USA). We could not obtain further information on these samples.

\section{Plasma preparation}

The blood samples were drawn in EDTA or citrated anticoagulant tubes. The plasma samples were prepared according to the platelet-rich procedure and stored at $-80^{\circ} \mathrm{C}$ until use. Prior to the assay, plasmas were thawed at $37^{\circ} \mathrm{C}$ for 15 minutes and centrifuged at $1,500 \times g$ for 5 minutes.

\section{Antibody conjugation to magnetic beads}

The 3E7 anti-prion monoclonal antibody (Roboscreen Inc., Leipzig, Germany) was conjugated to tosyl-activated magnetic beads according to the manufacturer's instructions
(Invitrogen, Waltham, MA, USA) 3E7 was chosen because each stock obtained from the manufacturer demonstrates consistent activity between analyses. Anti-prion monoclonal antibodies such as 3E7 and T2 contained overlapping epitopes, meaning that the epitopes would be in competition with each other. The epitope of 3E7 is between 138 and 144 and 150 and 155 in reference to human and bovine sequences, respectively. The epitope of T2 is between 135 and 140 and 137 and 142 in reference to human and bovine sequences, respectively. The antibodies $3 \mathrm{E} 7$ and T2 were conjugated on the magnetic particles and horse radish peroxidase (HRP) as capture and detection antibodies, respectively. Since T2 was a Fab2 fragment, T2 would not be the best antibody to be conjugated to magnetic particles due to instability. The antibody 3E7 $(80 \mu \mathrm{g})$ was conjugated through tosyl-activated conjugation moiety on the magnetic particle (Dynabeads M-280, $2.8 \mu \mathrm{m}$ size). T2 was preconjugated by Dr Yokoyama's group at NIAH. Many prion antibodies were screened by MDS, and several antibodies were species specific (results not shown). Set of 3E7 and T2-HRP provided best results. Western blot and IHC tests would involve sample denaturation, which would open up the epitopes for those and other antibodies. MDS did not denature the samples; instead, it detects available epitopes after the sample dilution.

\section{Multimer detection system}

Plasma $(400 \mu \mathrm{L})$ was diluted with $1 \%$ Triton X-100 in Trisbuffered saline and Tween 20 (TBST) to $1.4 \mathrm{~mL}$ in a $2 \mathrm{~mL}$ tube. The addition of premixed capture and detection antibody cocktail (3E7-magnetic beads $[2 \mu \mathrm{L}$ in total of $\sim 0.8 \mu \mathrm{g}$ of beads] and T2-HRP [0.8 $\mu \mathrm{g}]$ in TBST) to the sample tube initiated the MDS assay. The ratio of two antibodies used in the cocktail was $1: 1$. The samples were mixed and incubated for 1 hour with rotation at $37^{\circ} \mathrm{C}$. The antibody and antigen complex was then concentrated using a magnet (Invitrogen). The supernatant and unbound complexes were removed, and the magnetic beads were washed with TBST. After transferring the magnetic beads to the microtiter plate, the Super Signal ELISA Pico chemiluminescent substrate (150 $\mu \mathrm{L}$; Thermo Fisher Scientific Inc., Waltham, MA, USA) was added, and the developed signal was quantified with the PerkinElmer Victor 3 (Waltham, MA, USA) multi-spectrophotometer for luminescence detection.

\section{Results}

\section{Clinical samples}

Figure 2 shows the relative luminescence (relative luminescence unit [RLU]) by MDS analysis for scrapie plasma 


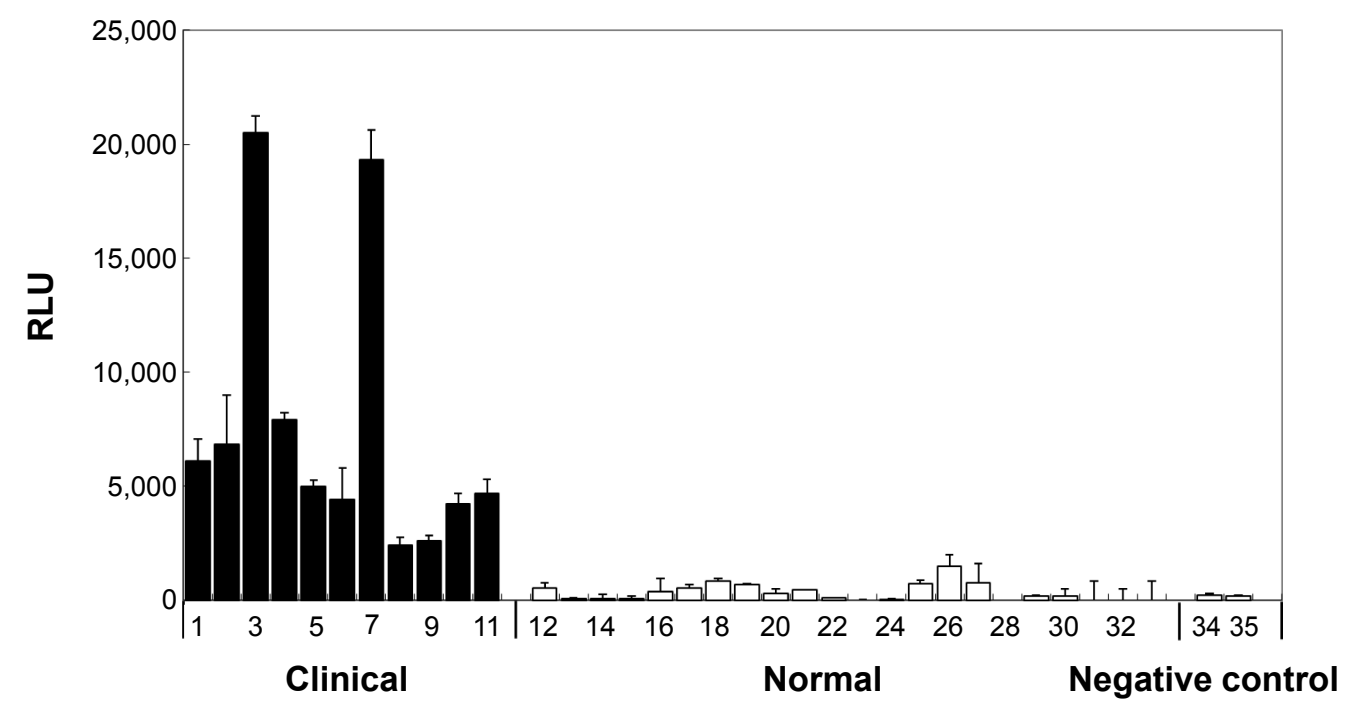

Figure 2 Differentiating scrapie sheep plasmas with clinical symptoms.

Notes: Scrapie sheep were well characterized by immunohistochemistry analyses of the third eyelid, tonsil, mand, retro, mess, and brain. Plasma samples from these scrapie sheep were tested by MDS, and all samples demonstrated higher RLUs than all of the normal healthy controls. The normal healthy control plasmas were drawn from sheep without any neurological symptoms. Three scrapie plasma samples were drawn from sheep, which were experimentally inoculated through the intracranial route with scrapie brain homogenate. The rest of the scrapie plasma samples were from naturally infected sheep. Test was performed in duplicate.

Abbreviations: MDS, multimer detection system; RLUs, relative luminescence units.

samples (\#1-11) and normal samples (\#12-33). All of the samples from the naturally or experimentally infected sheep demonstrated significantly higher RLUs than all the normal samples. The average RLUs for scrapie and normal samples were $7,630 \pm 6,290$ and $330 \pm 382$, respectively ( $P$-values obtained from a two-sided test were $<0.0001$; $P(T \leq t)=0.000017)$. Therefore, $100 \%$ sensitivity (eleven positive out of eleven scrapie samples) and $100 \%$ specificity (22 negative out of 22 healthy samples) were demonstrated by the MDS analysis in these limited samples. Negative controls consisted of using the following samples without addition of the detection antibody (T2-HRP): control samples (\#34, \#35), \#4 (BREF pooled scrapie), and \#20 (NIAH normal). A blank measurement with only the chemiluminescent substrate was taken as background, and this value was subtracted from all sample values. All measurements were performed in duplicate.

\section{Preclinical scrapie plasma samples}

Clinical signs were not observed in any of the 8-month-old preclinical sheep, and $\mathrm{PrP}^{\mathrm{Sc}}$ were detectable in the tonsil tissues of only a few animals. From the ten preclinical samples, MDS detected PrP multimers in five samples. Low RLUs similar to those of the eight age-matched normal control samples were observed in the other five preclinical samples. These results indicate that in these samples, MDS demonstrated $50 \%$ sensitivity and $100 \%$ specificity (Figure 3 ).

\section{Coded test with preclinical scrapie plasma samples}

A coded study comparing 12 plasma samples from scrapie sheep with clinical symptoms to those of 21 normal healthy controls was performed (Figure 4). These plasma samples were obtained from well-characterized clinical sheep. The genotype of scrapie clinical samples C1, C5, C6, C9, C11, and $\mathrm{C} 12$ was $A R Q / V R Q$, and that of the samples $\mathrm{C} 2, \mathrm{C} 3$, $\mathrm{C} 4, \mathrm{C} 7, \mathrm{C} 8$, and $\mathrm{C} 10$ was VRQ/VRG. The genotype of the majority of normal healthy samples was VRQ/VRQ, whereas N6, N11, N16, N17, and N21 were of the ARQ/VRQ genotype. All clinical scrapie samples along with normal control samples were coded independently and tested by MDS. After acquiring the data, the key was opened and analyzed. MDS detected eleven scrapie samples out of 12 (except C12). No false-positive results were obtained, although a slightly high RLU was observed in the N10 sample. This resulted in $91.7 \%$ sensitivity and $100 \%$ specificity in these samples.

Another panel of seven preclinical and 15 normal samples was coded and tested with MDS (Figure 5). Six out of seven preclinical samples were tested as positive by MDS, yielding $85.7 \%$ sensitivity and $100 \%$ specificity. The false-negative scrapie sample was pretreated with protease, suggesting that $\mathrm{PrP}^{\mathrm{Sc}}$ in the blood were protease sensitive, and the TSE diagnosis with protease might not accurately detect a scrapie sample. These two panels of blinded studies clearly differentiated scrapie samples from normal samples 


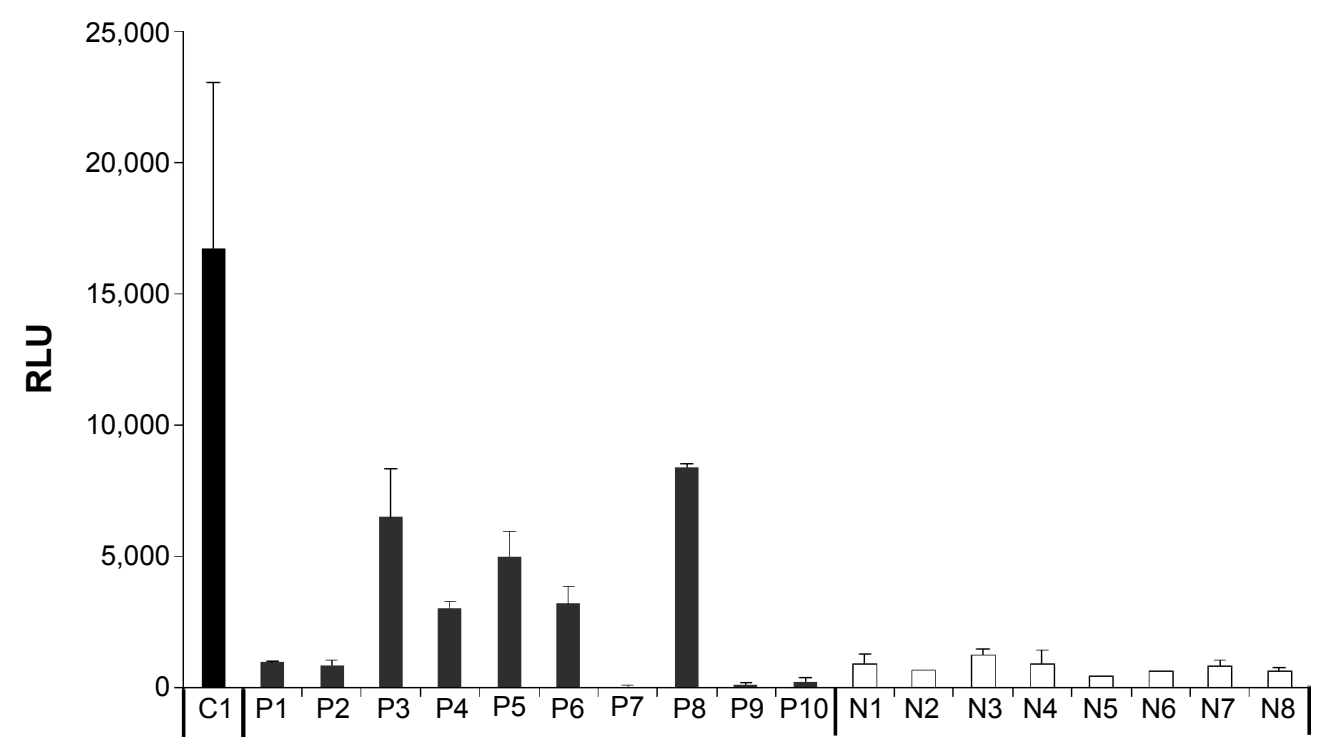

\section{Clinical Preclinical Normal}

Figure 3 Differentiating preclinical scrapie sheep plasmas in comparison to age-matched normal healthy controls.

Notes: These sheep with the VRQ/VRQ genotype were from a well-characterized control study of natural infection through birth. Both the ewe and lamb of these sheep died of scrapie after $\sim 22$ months. Blood was drawn from these preclinical scrapie sheep at the age of 8 months with citrated anticoagulant. These sheep also died of scrapie after 22 months. MDS accurately detected the presence of PrPsc in the blood of five out of ten animals. Test was performed in duplicate.

Abbreviations: MDS, multimer detection system; RLU, relative luminescence unit; $\mathrm{PrPsc}$, infectious prion proteins.

by MDS, suggesting the high specificity of MDS for detecting aggregated $\mathrm{PrPSc}^{\mathrm{S}}$.

\section{Discussion}

In an effort to prevent the spread of prion diseases through blood transfusion, leukoreduction filters were used, which significantly reduced exogenous and endogenous prion infectivity in red blood cell preparations made using scrapie-infected hamster blood for transfusion. ${ }^{19}$ Prion infectivity in the blood was suggested to be associated with circulating lymphocytes in the blood, the lymph, and tissues. ${ }^{20,21}$ Furthermore, lymph nodes were also suggested to function as major exchange sites for the recirculating lymphocytes. Therefore, leukoreduction was proposed to reduce the risk of vCJD transmission through blood transfusion in humans. ${ }^{13,23}$ However, leukoreduction could not prevent BSE transmission through

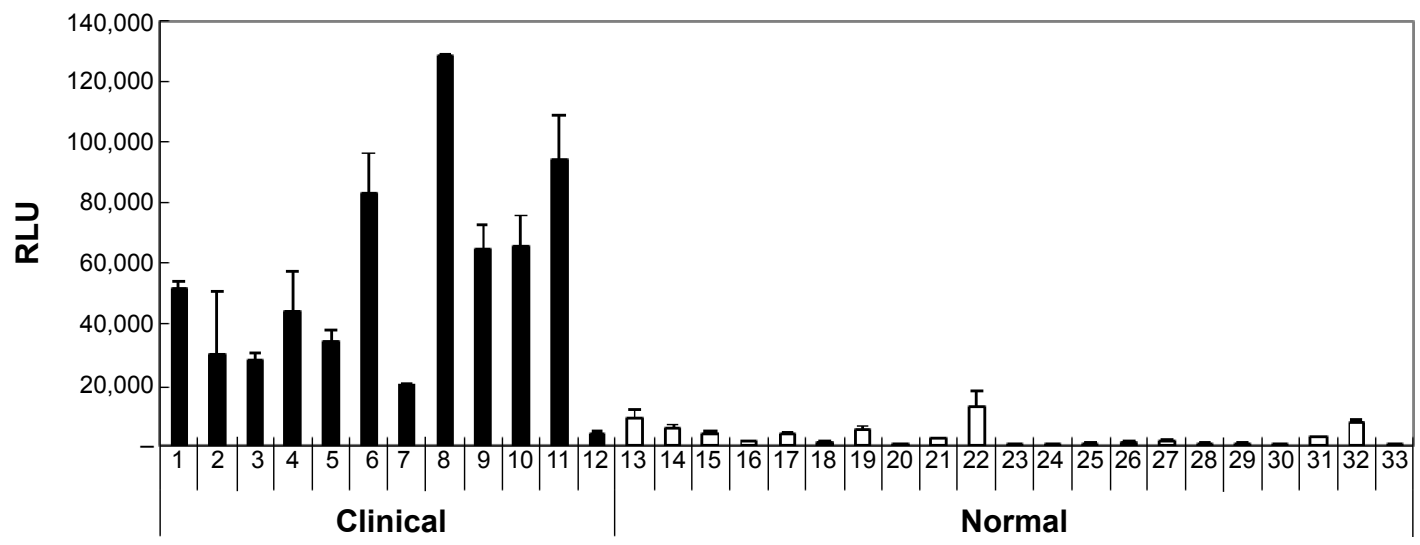

Figure 4 Coded study using limited plasma from sheep with clinical symptoms compared to normal healthy controls.

Notes: Plasma samples for this analysis were donated from well-characterized clinical sheep. Citrate was used as an anticoagulant. All clinical scrapie samples along with normal control samples were coded independently and tested by MDS. After acquiring the data, the key was opened and analyzed. The genotype of scrapie clinical samples $\mathrm{Cl}, \mathrm{C5}, \mathrm{C6}, \mathrm{C}$, $\mathrm{CI}$, and $\mathrm{Cl} 2$ was ARQ/VRQ, and that of samples $\mathrm{C} 2, \mathrm{C} 3, \mathrm{C} 4, \mathrm{C7}, \mathrm{C8}$, and $\mathrm{Cl} 0$ was VRQ/VRG. The genotype of the majority of normal healthy samples was VRQ/VRQ, whereas N6, NII, NI6, NI7, and N2I were of the ARQ/VRQ genotype. We could not obtain further information on these samples. Test was performed in duplicate.

Abbreviations: MDS, multimer detection system; RLU, relative luminescence unit. 


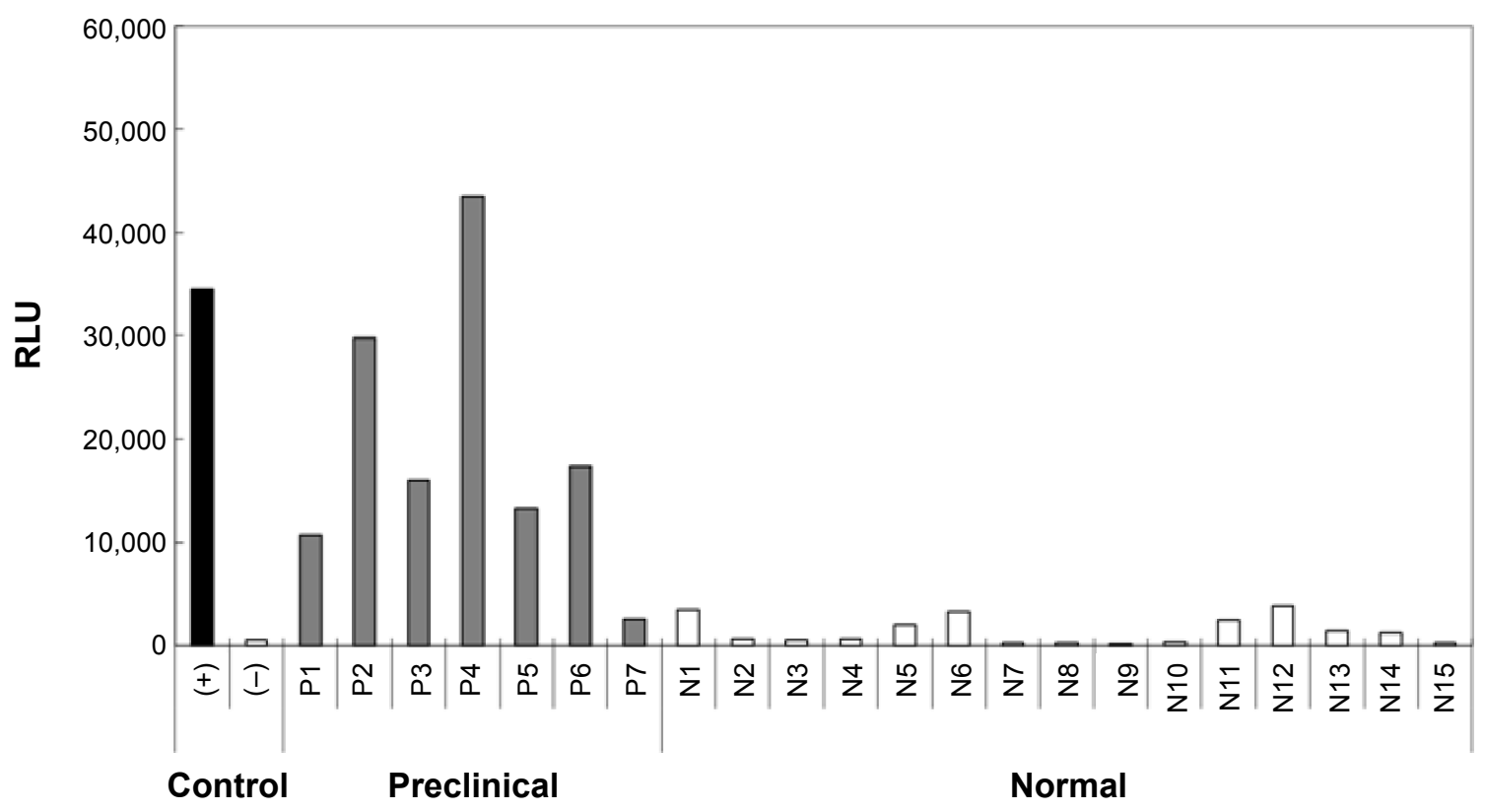

Figure 5 Coded study with limited preclinical sheep plasma without clinical symptoms compared to normal healthy controls.

Notes: These plasma samples were donated from well-characterized preclinical sheep. Citrate was used as an anticoagulant. All preclinical scrapie samples along with normal control samples were coded independently and tested by MDS. After acquiring the data, the key was opened and analyzed. We could not obtain further information on these samples. Test was performed in singlet.

Abbreviations: MDS, multimer detection system; RLU, relative luminescence unit.

single blood transfusion in sheep. ${ }^{23}$ In several studies, prion infectivity was extensive in sheep blood, and was shown to be present in the plasma, whole blood, and buffy coat. ${ }^{12,24-26}$ Interestingly, volumes of $50-135 \mathrm{~mL}$ of whole blood from scrapie-infected sheep were enough to induce transmission. Therefore, detection of prion infectivity with small volumes of blood would be beneficial for blood-based TSE diagnostic tests. Here, we used MDS to detect $\operatorname{PrP}^{\mathrm{Sc}}$ in a small volume $(400 \mu \mathrm{L})$ of plasma from scrapie-infected sheep that were confirmed positive for scrapie in postmortem analyses of the brain and other lymphoid tissues.

One obvious difference between monomers and multimers is that the unique epitope in the monomeric protein would exist in multiple copies in the multimeric forms. MDS utilized this multimeric feature of disease-associated proteins by distinguishing TSE-affected animals from healthy controls using their blood, without the need for a PMCA reaction assay. MDS resembles a traditional sandwich ELISA, except that the capture and detection antibodies are directed against the overlapping epitope for competition. Samples are prepared by a simple step involving buffer dilution, without PK digestion. This is followed by the initiation of the MDS procedure, which includes only one incubation step involving concurrent addition of the capture and detection antibodies for the detection of multimers. As a consequence, the unique epitope on the monomer becomes occupied by either a capture or detection antibody, not by both, which shields it from further reaction. Conversely, the multimeric forms of $\mathrm{PrP}^{\mathrm{Sc}}$ offer multiple epitopes for both capture and detection antibodies to bind simultaneously, resulting in stronger signals from samples with multimers than from monomer-containing samples. Therefore, MDS is capable of detecting multimers with high specificity in one step without the need for protease digestion. We do not completely understand the nature of the conformation of the multimeric forms of $\mathrm{PrP}^{\mathrm{Sc}}$ detected by MDS in this study. However, a previous study demonstrated that these multimers are not found in the brain or plasma of healthy hamsters. MDS previously differentiated various samples of scrapie-infected hamster brain homogenate, plasma spiked with brain homogenate from a TSE-infected mouse, and scrapie-infected hamster plasmas from corresponding normal samples. Based on previous reports, ${ }^{27,28}$ the infectivity observed in sheep plasma may or may not be due to the protease-resistant $\operatorname{PrP}^{\mathrm{Sc}}$. Since the relationship between infectivity and resistance or sensitivity of $\mathrm{PrP}^{\mathrm{Sc}}$ is not clearly defined, it is now easy to determine the nature of the material in the plasma. The findings from previous studies suggest that 1) $\mathrm{PrP}^{\mathrm{Sc}}$ exists in the blood of TSE-affected sheep and 2) MDS is capable of differentiating blood from scrapie-infected animals from that of normal healthy controls and from sheep at the preclinical stage. The reason for testing highly susceptible VRQ/VRQ genotype for typical, 
classical scrapie was to demonstrate the proof of concept of MDS in detecting the prion multimers in the typical, classical scrapie sheep, which were thoroughly studied. It would be very interesting to continue the comparative study with other genotypes in future.

In the current study using plasma from sheep at clinical and preclinical stages, MDS detected abnormal PrP with high sensitivity and specificity. In clinical stage scrapie-infected sheep, MDS accurately differentiated eleven scrapie-infected sheep from 22 normal sheep. In preclinical stage lambs, MDS revealed the presence of scrapie multimers in five out of ten animals, and all eight negative samples were correctly diagnosed. It is unknown whether all the scrapie-infected sheep had endogenous prions at low levels below our detection in their blood. The ten naturally infected lambs showed no symptoms at the preclinical stage. One of the blinded studies presented herein was performed with 12 scrapie clinical (C1-12) and 21 normal (N1-21) samples. MDS clearly differentiated scrapie samples from normal healthy samples in this analysis, except for one C12 scrapie sample. Finally, MDS correctly identified 22 out of 22 individual sheep blood samples in a coded panel. Among the 22 samples, 15 and seven were from normal and asymptomatic preclinical sheep, respectively. No false positives were identified from MDS (100\% specificity) in all clinical, preclinical, and coded panels.

Considering that blood collection is the least invasive method for identifying infected animals or humans, MDS could offer substantial advantages over other current methods. In the current study, MDS detected $\mathrm{PrP}^{\mathrm{Sc}}$ in sheep plasma, as early as 8 months. This raises the possible application of MDS in high-throughput screening of prion diseases in humans and animals. The sensitivities of MDS can possibly be influenced by in vitro and in vivo factors, and the sensitivities observed in the current samples may not differ from other prion samples.

Further studies will be needed to determine whether MDS can be used to detect naturally occurring diverse PrP strains of varied genotypes, such as familial PrP mutations. The variability of the MDS assay for different prion sample types should also be addressed, and adjustments for each individual species should be made as necessary. However, the basic principles of MDS should still be applicable across species. There may be unforeseen challenges for MDS when it is applied more widely. For example, other non-PrP amyloids may react and block $\mathrm{PrP}^{\mathrm{Sc}}$ detection.

Screening infectious prions in the preclinical stage by MDS using blood could help in discriminating and reducing the spread of these diseases. The first report of the earliest detection of $\mathrm{PrP}^{\mathrm{Sc}}$ was done in blood from 8-month-old lambs. ${ }^{29}$ Depending on the genotypes, sheep normally show symptoms of scrapie at 2-5 years of age. Identification of infected sheep 1-4 years prior to the manifestation of symptoms will allow effective removal of infected animals before they can transmit scrapie to healthy sheep in the flock.

The platelet-rich plasma fraction from scrapie-infected sheep was used in MDS. Platelets or platelet-rich plasma pellets in white-tailed deer ${ }^{30}$ and sheep ${ }^{23}$ were shown to contain chronic wasting disease (CWD) and BSE infectivity, respectively. Platelets from scrapie-infected hamsters were not able to transmit scrapie; ${ }^{31}$ however, $\mathrm{PrP}^{\mathrm{Sc}}$ in plateletfree hamster plasma samples were detected by PMCA. ${ }^{32,33}$ Other studies also revealed that $\mathrm{PrP}^{\mathrm{Sc}}$ infectivity exists in the cell-free plasma of sheep. ${ }^{34,35}$ These findings suggest the presence of varying infectivity in rodents and ruminants. Interestingly, highly purified radioactively labeled murine $\mathrm{PrPSc}^{\mathrm{Sc}}$ could pass through the mouse blood-brain barrier, ${ }^{36}$ suggesting the possible existence of cell-free $\mathrm{PrP}^{\mathrm{Sc}}$ in the blood.

vCJD infectivity in plasma components was reported in a hemophilic recipient, who was exposed to cell-free fractionated plasmas. ${ }^{37,38}$ Depending on the titer of transmitted $\mathrm{PrP}^{\mathrm{Sc}}$, the detection of preclinical scrapie in recipient lambs may take anywhere from between 594 days and 1,089 days posttransmission. CWD infectivity in white-tailed deer was not found in platelet-poor plasma but in the platelets, whereas infectivity was observed in the plasma of sheep, hamsters, and humans. ${ }^{30}$ These results demonstrate that MDS could identify endogenous prions, which could be applied to a vCJD diagnostic blood test.

\section{Conclusion}

MDS, which is built on a simple and very straightforward concept, offers all the advantages and sensitivity in detecting $\mathrm{PrP}^{\mathrm{Sc}}$ from preclinical sheep. The main merits of MDS were that it was developed to provide signals from only samples containing prion multimers among high concentrations of monomers in one-pot reaction. Sample, 3E7 capture antibody, and T2-HRP detection antibody should be mixed in one reaction tube for efficient detection. It also offers reproducibility, specificity, rapidity, simplicity for routine use, and amenability to automation in human blood screening to ensure the safety of blood and blood products. MDS could also be a tool for screening inhibitors against oligomerization, monitoring potential drug efficacy, and early 
diagnosis before the onset of extensive damage from $\mathrm{PrP}^{\mathrm{Sc}}$. In addition, since MDS was a detection system, it could be combined with PMCA or real-time quaking-induced conversion to increase sensitivity and reduce the amplification time. Lastly, monitoring the presence of multimers by MDS appears to be particularly useful for other protein misfolding conformational diseases, such as Alzheimer's, Huntington's, and Parkinson's. MDS could also be used to diagnose other similar neurodegenerative protein diseases.

\section{Acknowledgments}

This research was supported by grants from the Gachon University (GCU-2014-0190) and National Research Foundation of Korea (NRF-2010-0024004). The authors would like to thank Ervin Welker from the Hungary Academy of Sciences (Budapest, Hungary) for providing normal sheep samples.

\section{Disclosure}

The authors report no conflicts of interest in this work.

\section{References}

1. Béringue V, Herzog L, Jaumain E, et al. Facilitated cross-species transmission of prions in extraneural tissue. Science. 2012;335:472-475.

2. Quadrio I, Ugnon-Café S, Dupin M, et al. Rapid diagnosis of human prion disease using streptomycin with tonsil and brain tissues. Lab Invest. 2009;89:406-413.

3. Rodríguez-Martínez AB, Garrido JM, Zarranz JJ, et al. A novel form of human disease with a protease-sensitive prion protein and heterozygosity methionine/valine at codon 129: case report. BMC Neurol. 2010; 10:99.

4. Safar JG. Molecular pathogenesis of sporadic prion diseases in man. Prion. 2012;6:108-115.

5. Van Dorsselaer A, Carapito C, Delalande F, et al. Detection of prion protein in urine-derived injectable fertility products by a targeted proteomic approach. PLoS One. 2011;6:e17815.

6. Lourenco PC, Schmerr MJ, MacGregor I, Will RG, Ironside JW, Head MW. Application of an immunocapillary electrophoresis assay to the detection of abnormal prion protein in brain, spleen and blood specimens from patients with variant Creutzfeldt-Jakob disease. J Gen Virol. 2006;87:3119-3124.

7. Murayama Y, Yoshioka M, Horii H, et al. Protein misfolding cyclic amplification as a rapid test for assessment of prion inactivation. Biochem Biophys Res Commun. 2006;348:758-762.

8. Bellon A, Seyfert-Brandt W, Lang W, Baron H, Gröner A, Vey M. Improved conformation-dependent immunoassay: suitability for human prion detection with enhanced sensitivity. J Gen Virol. 2003;84:1921-1925.

9. Silveira JR, Hughson AG, Caughey B. Fractionation of prion protein aggregates by asymmetrical flow field-flow fractionation. Methods Enzymol. 2006;412:21-33.

10. Colby DW, Prusiner SB. Prions. Cold Spring Harb Perspect Biol. 2011; 3:a006833.

11. Menache D, O'Malley JP. Creutzfeldt-Jakob disease: current U.S. policy to further reduce the possible risk of transmission by transfusion. Biologicals. 1996;24:277-283.

12. Houston F, McCutcheon S, Goldmann W, et al. Prion diseases are efficiently transmitted by blood transfusion in sheep. Blood. 2008; 112:4739-4745
13. Cervia JS, Sowemimo-Coker SO, Ortolano GA, Wilkins K, Schaffer J, Wortham ST. An overview of prion biology and the role of blood filtration in reducing the risk of transfusion-transmitted variant CreutzfeldtJakob disease. Transfus Med Rev. 2006;20:190-206.

14. Hilton DA, Ghani AC, Conyers L, et al. Prevalence of lymphoreticular prion protein accumulation in UK tissue samples. J Pathol. 2004;203: 733-739.

15. Race R, Raines A, Raymond GJ, Caughey B, Chesebro B. Long-term subclinical carrier state precedes scrapie replication and adaptation in a resistant species: analogies to bovine spongiform encephalopathy and variant Creutzfeldt-Jakob disease in humans. J Virol. 2001;75:10106-10112.

16. Orrú CD, Wilham JM, Raymond LD, et al. Prion disease blood test using immunoprecipitation and improved quaking-induced conversion. M Bio. 2011;2:e00078-e11.

17. An SS, Lim KT, Oh HJ, et al. Differentiating blood samples from scrapie infected and non-infected hamsters by detecting disease-associated prion proteins using Multimer Detection System. Biochem Biophys Res Commun. 2010;392:505-509.

18. Andréoletti O, Berthon P, Marc D, et al. Early accumulation of $\operatorname{PrP}(\mathrm{Sc})$ in gut-associated lymphoid and nervous tissues of susceptible sheep from a Romanov flock with natural scrapie. J Gen Virol. 2000; $81: 3115-3126$

19. Sowemimo-Coker S, Kascsak R, Kim A, et al. Removal of exogenous (spiked) and endogenous prion infectivity from red cells with a new prototype of leukoreduction filter. Transfusion. 2005;45: 1839-1844.

20. Young AJ, Dudler L, Yamaguchi K, Marston W, Hein WR. Structure and expression of ovine complement receptor type 2. Vet Immunol Immunopathol. 1999;72:67-72.

21. Pan C, Baumgarth N, Parnes JR. CD72-deficient mice reveal nonredundant roles of $\mathrm{CD} 72$ in B cell development and activation. Immunity. 1999;11:495-506.

22. Hewitt PE, Llewelyn CA, Mackenzie J, Will RG. Creutzfeldt-Jakob disease and blood transfusion: results of the UK Transfusion Medicine Epidemiological Review study. Vox Sang. 2006;91:221-230.

23. McCutcheon S, Alejo Blanco AR, Houston EF, et al. All clinicallyrelevant blood components transmit prion disease following a single blood transfusion: a sheep model of vCJD. PLoS One. 2011; 6:e23169.

24. Houston F, Foster JD, Chong A, Hunter N, Bostock CJ. Transmission of BSE by blood transfusion in sheep. Lancet. 2000;356: 999-1000

25. Hunter N, Foster J, Chong A, et al. Transmission of prion diseases by blood transfusion. J Gen Virol. 2002;83:2897-2905.

26. Dassanayake RP, Schneider DA, Truscott TC, Young AJ, Zhuang D, O'Rourke KI. Classical scrapie prions in ovine blood are associated with B lymphocytes and platelet-rich plasma. BMC Vet Res. 2011;7:75.

27. Shaked GM, Shaked Y, Kariv-Inbal Z, Halimi M, Avraham I, Gabizon R. A protease-resistant prion protein isoform is present in urine of animals and humans affected with prion diseases. J Biol Chem. 2001;276:31479-31482.

28. Kuczius T, Groschup MH. Differences in proteinase K resistance and neuronal deposition of abnormal prion proteins characterize bovine spongiform encephalopathy (BSE) and scrapie strains. Mol Med. 1999;5: 406-418.

29. Jackman R, Everest DJ, Schmerr MJ, Khawaja M, Keep P, Docherty J. Evaluation of a preclinical blood test for scrapie in sheep using immunocapillary electrophoresis. $J$ AOAC Int. 2006;89:720-727.

30. Mathiason CK, Hayes-Klug J, Hays SA, et al. B cells and platelets harbor prion infectivity in the blood of deer infected with chronic wasting disease. $J$ Virol. 2010;84:5097-5107.

31. Holada K, Vostal JG, Theisen PW, MacAuley C, Gregori L, Rohwer RG. Scrapie infectivity in hamster blood is not associated with platelets J Virol. 2002;76:4649-4650.

32. Murayama Y, Yoshioka M, Okada H, Takata M, Yokoyama T, Mohri S Urinary excretion and blood level of prions in scrapie-infected hamsters J Gen Virol. 2007;88:2890-2898. 
33. Tsukui K, Takata M, Tadokoro K. A potential blood test for transmissible spongiform encephalopathies by detecting carbohydrate-dependent aggregates of PrPres-like proteins in scrapie-Infected hamster plasma. Microbiol Immunol. 2007;51:1221-1231.

34. Rubenstein R, Chang B, Gray P, et al. A novel method for preclinical detection of PrPSc in blood. J Gen Virol. 2010;91:1883-1892.

35. Banks WA, Niehoff ML, Adessi C, Soto C. Passage of murine scrapie prion protein across the mouse vascular blood-brain barrier. Biochem Biophys Res Commun. 2004;318:125-130.
36. Siso S, Jeffrey M, Gonzalez L. Neuroinvasion in sheep transmissible spongiform encephalopathies: the role of the haematogenous route. Neuropathol Appl Neurobiol. 2009;35:232-246.

37. Hewitt P. vCJD and blood transfusion in the United Kingdom. Transfus Clin Biol. 2006;13:312-316.

38. Lefrere JJ, Hewitt P. From mad cows to sensible blood transfusion: the risk of prion transmission by labile blood components in the United Kingdom and in France. Transfusion. 2009;49:797-812.

\section{Publish your work in this journal}

The International Journal of Nanomedicine is an international, peerreviewed journal focusing on the application of nanotechnology in diagnostics, therapeutics, and drug delivery systems throughout the biomedical field. This journal is indexed on PubMed Central, MedLine, CAS, SciSearch $®$, Current Contents ${ }^{\circledR} /$ Clinical Medicine,
Journal Citation Reports/Science Edition, EMBase, Scopus and the Elsevier Bibliographic databases. The manuscript management system is completely online and includes a very quick and fair peer-review system, which is all easy to use. Visit http://www.dovepress.com/ testimonials.php to read real quotes from published authors.

Submit your manuscript here: http://www.dovepress.com/international-journal-of-nanomedicine-journal 